\title{
Twenty Two Test Positive to Covid-19 at One Health Facility in one Month: Who, Where, How, What should be done?
}

\author{
Jordan Tembo ${ }^{1}$, Patricia Mambwe ${ }^{2}$ \\ ${ }^{1}$ Kalindawalo General Hospital, P.O.Box 560008, Petauke, Zambia \\ ${ }^{2}$ Department of Nursing, School of Health Sciences, Rusangu University, Monze, Zambia
}

\begin{abstract}
COVID-19 is a mysterious medical condition that has affected the entire global society. Health Workers face the greatest possible threat of contracting the infection and even dying out of it. At the beginning of winter, one health facility in Zambia recorded a total of 22 Health Workers infected with COVID-19 during the month of June, 2021. The objective of this inquiry was to establish where and how the staff acquired the infection. The study used all the 22 Health Workers with history of confirmed COVID-19 during the month of June. The study was conducted between June and July, 2021. Single-interviewper-participant data collection method was used to collect data from participants. Participation was voluntary. This study found that Health Workers on night duty in the COVID-19 isolation ward worked longer hours, got exhausted with subsequent increased exposure risk and eventual acquisition of infection through close physical contact with Covid-19 patients or contaminated Personal Protective Equipment. This study recommends that Ministry of Health to increase the number of Health Workers- nurses, doctors and other cadres essential in the provision of health care services to COVID-19 patients and to provide Health Workers with adequate supplies of PPEs. Local Hospital Management to reduce working hours for health workers especially those taking night duty in the COVID-19 ward.
\end{abstract}

Key words: COVID-19, Health Workers, risk, exposure

\section{INTRODUCTION}

Grom the time the 'Chinese authorities identified a new Type of corona virus- COVID-19 from a cluster of pneumonia cases in Wuhan city, Hubei Province, China' (World Health Organization, 2020:5), COVID-19 has been an existential threat to mankind. The virus has since gained foothold and rapidly engulfed the globe thwarting plans and events, devastating lives, tearing families apart, ripping livelihoods away and overwhelming health workers. The disease has challenged modern civilization, hijacked routines, changed the direction of societies and twisted the arm of science, rolling it back to its rudiments. COVID-19 has severely affected the world's economic, social and mental life, causing inconsolable grief and untold suffering everywhere. Individuals across societies, unacclimated to the permanent emergency of daily masking up are compelled to hide their faces away behind the mask or face COVID-19 excruciating illness and possible death.
Wave after wave of new COVID-19 infections have surged and pounded continents mercilessly not sparing colour, race, ethnicity, gender and occupation. In Zambia, the first cases of COVID-19 were identified on 18th March, 2020 (Chipimo et al. 2020). Health Workers, the treasured 'frontline soldiers' against COVID-19, while fighting the deadly virus with utmost bravery and confidence contract the disease on duty (Pandey \& Sharma 2020: 939-942). A Health Worker is defined by the World Health Organization as all people engaged in actions whose primary intent is to enhance health (World Health Organization 2020).Included in this category are doctors, nurses, midwives, paramedical staff, hospital administrators, support staff and community Health Workers(Bandyopadhyay et al., 2020). Health workers face mortal danger like no other in the wake of fast-spreading COVID-19 infection. In a health facility, patients with fullblown or 'unusual symptoms of COVID-19 or very mild general flu-like symptoms pose considerable risk to healthcare workers who may not have a high level of clinical suspicion' (Chersich et al. 2020:2).

Health workers, face the greatest risk of contracting Corona virus because typically their work demands them to be in close physical proximity to patients and Health Workers working within a metre distance from COVID-19 patient were more likely to acquire the infection (Gamio 2020; Hussen \& Alemu, 2021). Kumar et al., 2020) observed that Health Workers working in or who visit crowded places often, or those who travel to many places such as restaurants or live in a confined space, have an increased possibility of transmitting (Kumar et al., 2020:140) or contracting Corona virus. Health workers, while on duty in the COVID-19 ward, wear Personal Protective Equipment (PPE) - face masks, gloves, gowns, goggles, face shield, head gear and shoe covers to prevent contamination of the hands, legs, skin, hair, eyes and clothes of healthcare workers that could lead to the transfer of infectious virus to the nose, mouth and eyes (European Centre for Disease Control Prevention and Control (ECDC) 2021:3). Healthcare workers are expected to work moderate hours and strictly follow the procedures for putting on and safely removing PPE in the correct sequence (ECDC, 2020; Ran et al., 2019). 
According to the World Health Organization (2020), between $25^{\text {th }}$ February and $4^{\text {th }}$ August, 2020, Zambia recorded 6, 580 COVID-19 infections. Out of this number, 115 were Health Workers (WHO 2020:4). Health workers working in Covid 19 ward and other critical departments are at a greater exposure risk of getting infected and even dying of COVID19. During the month of June, 2021 at one of the isolation health facilities in district Q (name deliberately withheld for confidentiality)- Zambia, 22 Health Workers out of 135 tested positive to COVID-19. What made it so alarming was that the month of June was the beginning of winter in Zambia. Wintry conditions covered the months of June to August. If $15.5 \%$ of the staff could test positive to COVID-19 within 4 weeks, then how many staff would test positive to COVID-19 at the end of winter if the trend continued? With Health Workeraccelerated Covid-19 infection at the health facility there was a looming threat of the virus 'spreading to colleagues, hospitalised patients and even family members' (Barraco \& Ventura 2020:66) threatening the facility of closure unless something was done to reduce the fast spreading Covid-19 infection. The objective of this inquiry was to establish where and how the staff acquired the infection. The study was conducted at a health facility in district Q- Zambia.

\section{Significance of the study}

The findings of this study will help to create awareness to policy makers to strengthen the Health System in Zambia.

\section{MATERIALS AND METHODS}

The study used an explorative design with a qualitative approach. It was conducted between June and July, 2021. Participants were purposively drawn from one health facility with an active covid-19 isolation ward in district Q. A total of 22 Health Workers with history of COVID-19 infection during the month of June, 2021 constituted the Total Population Sample (TPS) for the study. Respondents comprised 5 males and 17 females. Single-interview-perparticipant data collection method was used. Assurance was made to participants that the exercise was meant to learn where and how they got infected and grab their recommendations in order to prevent further transmission and re-infection. Data collection was conducted after participants recovered fully from COVID-19 infection. Interviews were conducted either in a face to face situation or on phone. Face to face interviews were conducted while observing all COVID-19 guidelines- masking, physical distancing and hand washing. Participants who had travelled and were out of physical reach, agreed for an interview through the phone at an appropriate time convenient to them. Each interview took 45-50 minutes. The interview was guided by an interview guide. Permission was granted to record conversations but to delete immediately after data analysis. From the scribbled notes, only numbers and not names or initials of participants were recorded to achieve anonymity and confidentiality. After data analysis, all the recorded audios related to the study were deleted and participants were called and informed about the deleting exercise. Qualitative data was analyzed through thematic analysis. The authors used thematic analysis for the purpose of 'generating new insights' (Braun \& Clarke, 2006:77-101) as no similar study had been conducted in a rural setting of Zambia. Data analysis followed a six-phase approach as outlined by Braun \& Clarke (2012) namely familiarising oneself with the data, generating initial codes, searching for themes, reviewing potential themes, defining and naming themes and producing report.

\section{RESULTS}

Table 1. Demographic characteristics of participants

\begin{tabular}{|c|c|c|c|c|c|c|c|}
\hline Variables & \multicolumn{3}{|c|}{ Participants under nursing/clinical } & \multicolumn{4}{|c|}{ Participants under administration } \\
\hline \multirow{2}{*}{ Gender } & Male & \multicolumn{2}{|l|}{ Female } & \multicolumn{2}{|l|}{ Male } & \multicolumn{2}{|l|}{ Female } \\
\hline & $4(18.1 \%)$ & \multicolumn{2}{|l|}{$12(54.5 \%)$} & \multicolumn{2}{|l|}{$3(13.6 \%)$} & \multicolumn{2}{|l|}{$3(13.6 \%)$} \\
\hline Age in years & $\begin{array}{ll}18-22 & 0 \\
23-27 & 0 \\
28-32 & 2 \\
33-37 & 1 \\
38-42 & 1 \\
43 \text { and above } & 0\end{array}$ & $\begin{array}{l}18-22 \\
23-27 \\
28-32 \\
33-37 \\
38-42 \\
43 \text { and above }\end{array}$ & $\begin{array}{l}0 \\
3 \\
6 \\
2 \\
0 \\
1\end{array}$ & $\begin{array}{l}18-22 \\
23-27 \\
28-32 \\
33-37 \\
38-42 \\
43 \text { and above }\end{array}$ & $\begin{array}{l}0 \\
0 \\
1 \\
2 \\
0 \\
0\end{array}$ & $\begin{array}{l}18-22 \\
23-27 \\
28-32 \\
33-37 \\
38-42 \\
43 \text { and above }\end{array}$ & $\begin{array}{l}0 \\
0 \\
2 \\
1 \\
0 \\
0\end{array}$ \\
\hline Highest Qualification & $\begin{array}{l}\text { Degree } \\
\text { Advanced Diploma } \\
\text { Diploma }\end{array}$ & $\begin{array}{c}\text { Degree } \\
\text { Advanced Diploma } \\
\text { Diploma }\end{array}$ & $\begin{array}{r}1 \\
1 \\
12\end{array}$ & $\begin{array}{l}\text { Degree } \\
\text { Advanced Diploma } \\
\text { Diploma } \\
\text { Grade } 12 \text { Cert. }\end{array}$ & $\begin{array}{l}1 \\
\mathrm{a} \\
0 \\
1 \\
1\end{array}$ & $\begin{array}{l}\text { Degree } \\
\text { Advanced Dip. } \\
\text { Diploma } \\
\text { Grade } 12 \text { Cert. }\end{array}$ & $\begin{array}{l}1 \\
0 \\
0 \\
2\end{array}$ \\
\hline
\end{tabular}

\section{Where do you think you acquired COVID-19 infection?}

This question was poised at investigating where COVID-19 infected Health Workers acquired the infection. They cited
CVID-19 isolation ward, workshop venue, own home, neighbour's houses, guest houses and restaurant as precise locations where they thought they acquired COVID-19 infection. 


\section{From COVID-19 isolation ward}

A total of 10 participants attributed the source of COVID-19 infection to the isolation ward. They narrated that contact with COVID-19 patients with full blown infection in the isolation ward potentially exposed them to the devastating virus. Their causal judgement affirmed that before commencing duty of seven days in the COVID-19 ward, they were full of bloom and physical exuberance. Immediately they immersed their toes in the COVID-19 isolation ward, a few days later, came a strange pushback feeling of un-wellness burrowing the head, muscles, appetite, and blunting the acute sense of smell and taste. In their narration, participants explained that they began to experience COVID-19 symptoms between the fifth to seventh day of duty. One female participant was quick to put a finger on the source of her COVID-19 infection. 'For me it's from the COVID-19 ward because five to six days into my duty in the ward, I began to feel unwell. My illness was gradual, gaining momentum in severity each day' (Female participant).

A male participant explained, 'I experienced a bone-crushing unpleasant feeling after working seven days at the COVID-19 isolation ward. I tried to ignore the mind-clouding feeling but it was so intense. I intuitively knew that if I took a COVID-19 test, the results would come out positive and sure it did' (Male participant). A female participant also traced her COVD-19 infection source to the isolation ward. 'I understand my body so well. I know how it reacts when something's wrong. My turn of duty at the isolation began pretty well. I was super fine but five days into my duty I began to feel entirely different... persistent headache and malaise muddened the waters of my physical and mental health. Immediately I went for a COVID19 swab. It came out positive' (Female participant).

\section{From the Antiretroviral clinic}

Two participants delineated the source of their COVID-19 infection from the antiretroviral clinic. One of them recounted her story, 'I acquired COVID-19 infection from the Antiretroviral Clinic' (Female participant). Her COVID chronicles began when an HIV positive client stepped in the clinic. Time taken to counsel the client amid coughs, exposed the participant to COVID-19 infection even if both had masked up. 'My client would pause to sneeze and cough. When the COVID-19 test was ordered, it was positive. Approximately six days after contact with the client, I began to experience severe backache. I felt as though my entire lower back would drop' (Female participant). The other female participant grafted her recount from the same storyline. 'I went to the antiretroviral clinic for data verification. I spent a sizeable amount of time at the clinic checking records with the staff in that unit. The next time I heard about her was that she was under self quarantine at home after testing positive to COVID-19. Three days later, I manifested with COVID-19 symptoms. Then I knew exactly where I got the infection. It was the antiretroviral clinic!' (Female participant).

\section{Workshop and meetings}

Three participants with a history of attending a workshop for 10 days explained of feeling physically subdued a couple of days into the activity. The trio explained that before attending the workshop, they felt physically whole. COVID19-associated symptoms pulled a surprise for them. A female participant admittedly said, 'I was away attending a workshop. Eight days into the workshop I was enjoying a blissful physical health. The ninth day hit me like a sledge hammer. I felt feverish and nauseated. I thought well this could be malaria. So I dashed to the nearest health facility. I was tested for Malaria and COVID-19. Guess the test that came out positive- COVID-19!' (Female participant). One of the male participants who had gone to attend a meeting from another district stated that, 'a day after I came back from the three days' meeting, I experienced unbearable chills that could resist cure with the warmest blanket' (Male participant). The male colleague reported that after the meeting, 'was over I remained behind for the weekend. I could not enjoy the weekend because I got very sick. I rushed to the hospital and was tested and the results showed I was positive to COVID19' (Male participant).

\section{Neighbour's House}

One female participant explained that her source of infection was the neighbour whom she had visited. 'I contracted COVID-19 from my neighbour' (Female participant). A day after her visit, she learnt that her neighbour tested positive to COVID-19. 'I was told that my neighbour tested positive to COVID-19'(Female participant). It was not a surprise for her to experience COVID-19 symptoms days later. 'When I began to experience similar symptoms as those described by my neighbour I knew I was infected as well' (Female participant). When she went for a COVID-19 test, it came out positive. The participant explained that she felt angry and disappointed with herself for her compliance with culture to visit sick neighbours. 'I felt angry and disappointed with myself for risking my life over cultural compliance to visit a neighbour' (Female participant).

\section{Guest house}

One participant explained, 'I acquired COVID-19 infection from the guest house' (Female participant). She was sharing a room with a suspected COVID-19 infected colleague. Her colleague would cough and sneeze in the night. She asked whether the colleague had gone for COVID-19 swabbing. The assumed negative answer was her precarious hope. 'When I asked my colleague whether she had taken a COVID-19 test she told me she tested negative' (Female Participant). She was skeptical. 'I did not believe her' (Female participant).

\section{Own home}

Five participants elucidated that they contracted COVID-19 from family members- husband, auntie and sister. One participant reported, 'for me COVID-19 was right in our home. My husband tested positive. It was not surprising at all 
for me to test positive a few days later' (Female participant). Another female participant explained that her husband- a teacher elsewhere experienced joint pains, fever and a headache. He went to the hospital believing he had malaria but was told he did not have malaria but covid. Seven days later I began to experience some chills. I could cover myself with several warm blankets without getting warm. The next day I rushed to the hospital and the covid swab came out positive' (Female participant). A male participant narrated that he had stopped over at his auntie's house in Lusaka. 'I found my auntie complaining of feeling feverish, muscle cramps and fatigue. I began to feel the same way six days later. I went for COVID-19 test and I tested positive to Covid' (Male participant). The third participant explained that he acquired covid while on leave in the capital city- Lusaka. 'I was away on leave in Lusaka. Then I noticed I could not pick the smell of kapenta. Then I went for a swab and whoops! It was positive' (Male participant).

\section{How did you acquire COVID-19 infection?}

This question was intended to elicit information on the way participants acquired COVID-19 infection. Responses from participants sifted through several layers of possibilities but finally circled contact with patients with a collection of symptoms of full-blown COVID-19 infection, asymptomatic patients, friends, colleagues, family members and neighbours.

Contact with patients manifesting symptoms of full-blown COVID-19 infection

A total of 9 participants explained that they acquired covid infection from patients with advanced infection they nursed in the Covid-19 isolation ward. Participants narrated that contact with COVID-19 patients in the middle of duty exposed them to huge amounts of virus, increasing their risk of contracting the infection. A female participant curated, 'The month of June was the worst. Each day, the moment I reported for duty at the isolation, I could find 20 Covid-19 patients all of them very ill and on Oxygen. My colleague and I could come into extreme contact with Covid-19 patients as we bathed them, fed them, gave them medication, changed their diapers and ensured they had Oxygen masks on all the time. To me, that was already a clear shoe-in, kind of an exposure situation' (Female participant). The other participant amplified the colleague's response, 'when you work in an environment where all is nothing but Covid-19 then your exposure risk is twenty times more than anyone else' (Male participant). Other participants unravelled an interesting equation concerning physical exhaustion and the ability of the staff's adherence to strict infection prevention protocols. 'You know a staff working in COVID-19 ward is extra careful immediately they report for work at the covid-19 ward but physical exhaustion decelerates adherence to strict infection prevention and control' (Female participant). The latter participant explained that due to physical exhaustion, bad practices that compromised infection prevention guidelines emerged inadvertently. 'When a staff gets physically exhausted, it's possible to remove the face mask because it feels uncomfortable and by so doing they expose themselves to raging infection' (Female participant).

The latter participant believed it was partly due to physical exhaustion that staff acquired COVID-19 infection. 'Imagine two nurses per shift caring for over 20 very ill COVID-19 patients the whole long night that's crazy!'(Female participant). A male participant said in collaboration, 'I strongly believe the work was overwhelming' (Male participant).

\section{Contact with contaminated Personal Protective Equipment}

One participant explained that they contracted Covid-19 infection through contact with contaminated Personal Protective Equipment in the doffing area- the area reserved for taking off of used Personal Protective Equipment- 'I think I contracted COVID-19 at doffing' (Female participant). Doffing is the term denoting the step-by-step process for taking off of the Personal Protective Equipment (PPE). The participant explained that taking off of the Personal Protective Equipment demanded adherence to strict step-by-step procedure. Deviation at any point increases the risk of acquiring infection from the contaminated Personal Protective Equipment. 'The moment one gets careless at doffing either due to exhaustion, hunger, stress or because one is in a hurry, they can infect themselves or infect others including colleagues or family members just anyone they come in contact with' (Male participant). The participant added, 'I still insist that I exposed myself to Covid-19 infection when removing the PPE. Weary and hungry after working the whole night, with my entire soul I craved to rest. I accidentally dubbed the inside of the gown that's transferring the infection to my uniform' (Female participant).

Contact with patients, colleagues, family members with mild to zero symptoms

The 12 participants who traced their covid-19 infection from miscellaneous sources explained that contact with patients manifesting with mild to no symptoms at all made them acquire infection. 'When I found my auntie complaining of fever and chills I ran around with her for treatment and in the process greatly exposed myself to the virus' (Male participant). The participant who explained that she acquired covid-19 infection from the neighbour narrated, 'when I stepped in the room where my neighbour was sleeping I observed that he was coughing. I had a mask on but the surfaces I believe were all contaminated. The first question to cross my mind was what exactly was I doing in a COVID-19 contaminated environment like it or not I was potentially exposing myself to the virus' (Female participant). The participant with history of sharing a room with a colleague attributed the source of her infection to her roommate, 'I shared a room with a colleague. She could cough the whole night. On the last day of our stay I told her to get a COVID-19 swab immediately she got back. The same evening she called me that she had tested positive to COVID-19. That time I had 
already began feeling sick. I knew who had infected me. It was my roommate and my colleague' (Female participant). The participant who reported to have had contracted COVID19 in the capital city of Lusaka- narrated that he had been on leave for two months, 'I contracted COVID while away in the capital city. I suspect I acquired it from friends whom I would hang out with. I could go to eating places without masking up' (Male participant).

What should be done to prevent more staff from getting infected?

This question was meant to find out participants' recommendations tailored at arresting the spread of covid-19 infection to staff to ensure they were safe for uninterrupted health care service provision to patients. Participants explained that increasing the number of staff working in the isolation ward per shift, intensive orientation training in infection prevention for new staff, refresher infection training for staff already working in the isolation ward, and dropping infection prevention messages at every small gathering coupled with adequate supply of protective clothing and staying at home when off duty were sufficient to reduce acquisition of Covid-19 infection among staff.

Increasing the number of staff working in the COVID-19 isolation ward per shift

Participants reported that covid-19 isolation ward required adequate number of staff per shift. They insisted that an inadequate number of staff against a huge number of very ill patients, created an environment where the staff worked under huge exhausting demand. The nurse patient ratio was severely affected. A male participant explained, 'nursing extremely ill covid-19 patients require total holistic nursing care. If one staff has to nurse dozens of severely ill COVID-19 patients alone then that's a crisis already' (Male participant). Other participants talked about interrupted focus when the staff's attention was scattered among so many critically ill patients. 'When the staff's focus is on fewer clients, the staff is likely to do her best in an efficient and effective way but when everyone around requires resuscitation and other intensive procedures then one or two staff against scores of very sick patients is insufficient and possibilities of getting infected or infecting other patients increases' (Female participant).

\section{Intensive orientation training in infection prevention for new staff}

Participants explained that while Health Workers were naturally expected to be conversant with infection prevention through their formal certified training received before graduation, covid-19 was essentially different, highly transmissible and fast-spreading. Participants emphasized that COVID-19 required intensive training- a totally air-tight training without any margin of error. They stated that new staff expected to work at covid-19 staff were expected to undergo an uncompromising training to enable them adopt new attitudes, behaviours against protecting themselves and others. 'I recommend that all new staff assigned to work at the isolation ward should be fully oriented before stepping their feet in the deadly zone. Covid-19 ward is not a trial and error ward' (Female participant). A male participant simply said, 'it is suicidal to have an unoriented new staff at covid-19 ward' (Male participant). A female participant explained, 'we should not cheat each other that because we have graduated with honours then immediately we should be taken to work in the covid-19 ward? Oh. No. That's gambling with life' (Female participant). A male participant added new insight to the study through his contribution. 'It's possible for staff in the Covid19 ward to infect each other as peers while doing handovers so for me orientation training should include how to conduct safe handovers' (Male participant).

Refresher infection prevention training for staff already working in the isolation ward

Regarding refresher infection prevention for staff already allocated in the COVID-19 ward, participants explained that complete change of attitude and behaviour does not occur overnight among learners. 'If you learn about infection prevention once it doesn't mean you have absorbed everything at once' (Male participant). Another male participant collaborated the latter's view point, 'learning is incremental' (Male participant). Participants insisted that staff required regular refresher courses in infection prevention every week. 'I would say possibly every week there has to be a refresher training in infection prevention to provide staff with an opportunity to marry what they experienced with theoretical knowledge' (Female participant). The other female participant reported, 'I was oriented once. Do you think I know much? I don't think so. I feel inadequate. I need more capacity building training on infection prevention and control for me to perform to the utmost to ensure patients, colleagues, my family, the community including myself let me just say all of us are safe from COVID-19' (Female participant).

\section{Adequate supplies of Protective Equipment}

Participants reported that Personal Protective Equipment supplies were expected to be adequate at all times. 'Staff need to be in protective gear each time they enter COVID ward' (Female participant). The other male participant also said, 'face masks, gloves let me say everything essential for protection against Covid is important' (Male participant). A female participant explained that working in the Covid-19 ward did not call for exhibition of casual juvenile behaviour. 'Working in the Covid ward requires full protection and strict adherence to all protocols. No casual, macho behavioural display in the ward else you will get infected' (Female participant).

\section{Dropping of COVID messages at every meeting}

Participants explained that it was possible for staff to feel less in danger with persistence of Covid-19 infection. Regular dropping of Covid-19 messages during meetings was one important strategy to serve keep staff's sensitivity against 
Covid-19 infection alive and heightened. In such situations, familiarity bred danger and danger claimed staff as victims. A male participant said, "when a threatening condition lingers longer, people begin to feel part of it and less in danger and it is not uncommon for staff to remove a facemask while on duty right in the covid ward claiming covid is here to stay so it's better to learn to live with it. It's important to enforce the messages in its reality daily' (Male participant).

\section{Staying at home when not on duty}

Participants explained that staying at home when not on duty was an important strategy for staff to avoid getting COVID19. Travelling to near and distant places including undue visitations increased the risk of acquiring COVID-19 infection. 'Staying at home when off duty is the best strategy to avoid getting sick' (Male participant). A female participant said, 'it's common among us to travel and visit family members but I think with the large shadow of COVID-19 hovering around, it's extremely dangerous to hop around like a restless kangaroo' (Female participant).

\section{DISCUSSION}

COVID-19 is a treasonous medical condition that has betrayed the entire world to social and economical woes. Health Workers hailed as 'soldiers' at the battle front, are at the greatest COVID-19 infection risk. The objective of this study was to find out where and how 22 Health Workers with confirmed COVID-19 infection acquired the infection during the month of June at one health facility in Zambia. This research found that some Health Workers contracted COVID19 infection from the COVID isolation ward through close physical contact with confirmed COVID-19 patients in the course of duty to bathe, feed, medicate, or change soiled linen. Hussen and Alemu (2021) in their study titled Risk of COVID19 Infection and Associated Factors Among Healthcare Workers: A Cross-Sectional Study at Eka Kotebe Treatment Center in Ethiopia also found that Health Workers who performed their duties at a distance within one meter of a COVID-19 patient were 21 times more likely to be exposed to COVID-19 infection than those who were not within one meter of a COVID-19 patient (Hussen \& Alemu 2021:17631772).Confirming these findings, one participant in this study said, 'certain caring procedures for critically ill COVID-19 patients demands the caregiver to get so close' (Male participant). This study also found that other participants acquired COVID-19 infection from contaminated Personal Protective Equipment while doffing in the isolation ward. Findings of this study are highly consistent with those of Ran et al., (2019) whose observation emphasize that transmission from patients to Health Workers usually follows contamination of the HCWs' hands after touching patients or clothes/ materials that carry infection. This study also found that Health Workers who intruded environments housing a COVID-19 patient contracted COVID-19 infection. The environments include low-risk departments in the hospital, home, guest house, and restaurant but had a COVID-19 patient with moderate to mild symptoms acquired COVID-19 infection. Hussen and Alemu(2021) also found that Health Workers who have direct contact with an environment in which a COVID-19 patient were 10 times more likely to be exposed to COVID-19 infection than those who did not have such direct contact (Hussen \& Alemu 2021: 1763-1772).

This study found that Health workers who had worked night duty(reported for duty at 6 O'clock in the evening and knocked off the following day at 07:30 O'clock in the morning) acquired COVID-19 infection than those who worked during day shift taking only $6.5 \mathrm{hrs}$ did not contract COVID-19 infection. These findings support the findings conducted in China where Health workers working long hours were more susceptible to COVID-19 Infection (Ran et al. 2019). Moderate work hours benefit the health and safety of CHWs, while prolonged work (> 10 hours/day) would possibly increase the risk of respiratory infections $(\mathrm{Li} \& \mathrm{Wu}$, 2016; Weaver et al., 2020 in Ran et al., 2019). One participant in this study said, 'night duty is so exhausting because of long working hours' (Male participant).

\section{CONCLUSION}

The world, while in its near -serene atmosphere, then came COVID-19 infection shaking social events, disrupting culture, locking up occupations and worst of all claiming a train of precious lives including the lives of Health Workers. Health Workers by nature of their work, face the greatest COVID-19 infection risk. This study found that Health Workers on night duty in the COVID-19 isolation ward worked longer hours, got exhausted with subsequent increased exposure risk and eventual acquisition of infection through close physical contact with Covid-19 patients or contaminated Personal Protective Equipment.

\section{RECOMMENDATIONS}

This study recommends that Ministry of Health to increase the number of Health Workers- nurses, doctors and other cadres essential in the provision of health care services to COVID-19 patients and to provide Health Workers with adequate supplies of PPEs. Local Hospital Management to reduce working hours for Health Workers especially those taking night duty in the COVID-19 isolation ward. Local health facility leadership to prioritize on-going capacity building trainings in infection prevention for all staff, and to develop a careful monitoring system for strict adherence to infection prevention protocols.

\section{REFERENCES}

[1] Bandyopadhyay, S., Baticulon, R.E., Kadhum, M., et al (2020). Infection and mortality of healthcare workers worldwide from COVID-19: a systematic review. BMJ Global Health 2020;5: e003097. doi:10.1136/ bmjgh-2020-00309

[2] Barraco, R. Ventura, F.(2020). Covid-19 and infection in healthcare workers: An emerging problem. Medico-Legal Journal 2020, vol 88(2) 65-66 sage pub.com/journal permissions DOI: $10.1177 / 0025817220923649$ journals.sagepub.com/home/mlij 
[3] Braun, V., Clarke, V.(2006). Using Thematic Analysis in Psychology. Qualitative Research in Psychology, 3(2), 77-101 http://dx.do:org/10.1191/1478088706qp06309

[4] Braun, V., Clarke, V.(2012). Thematic Analysis. In H.Cooper, P.M.Camic, D. Sher (Eds.), APA handbook of research methods in psychology, vol. 2 Research designs: Quantitative, qualitative, neuropsychological, and biological, 57-71 American Psychological Association https://doi.org/10.1037/13620-004

[5] Chersich, M.F., Gray, G. Fairlie, L., Eichbaum, Q., Mayhow, S., Allwood, B., English, R., Scorgie, F., Luchters, S., Simpson, G., Haghighi, M.M., Pham, M.D., Rees, H.(2020). Covid-19 IN Africa, care and protection for frontline Health Workers. Globalization and Health https://doi.org/10.1186/s12992-02000574-3

[6] Chipimo, P.J., Barradas, D., Kayeyi, N, et al. (2020). First 100 persons with COVID-19-Zambia, March 18-April 28, 2020. MMWR Morb Mortal Wkly Rep 2020; 69: 1547-48

[7] European Centre for Disease Prevention and Control ECDC)(2021). Infection Prevention and Control and Preparedness for COVID-19 in healthcare settings Sixth update- $9^{\text {th }}$ February, 2021

https://www.ecdc.europa.eu/sites/default/files/documents/Infection -prevention-and-control-in-healthcare-settings-COVID19_6th_update_9_Feb_2021.pdf

[8] European Centre for Disease Prevention and Control $(E C D C)(2020)$. Guidance for wearing and removing personal protective equipment in healthcare settings for the care of patients with suspected or confirmed COVID-19.[cited 202102 August]. Available from: https://www.ecdc.europa.eu/en/publicationsdata/guidancewearing-and-removing-personal-protectiveequipment-healthcare-settings
[9] Gamio, L.(2020). The Workers Who Face the Greatest Corona Virus Risk. The New York Times $15^{\text {th }}$ March, 2020

[10] Hussen, H., Alemu, Z.A.(2021). Risk of COVID-19 Infection and Associated Factors Among Health Care Workers: A crosssectional study at Eka Katebe Treatment Centre in Ethiopia. International Journal of General Medicine vol. 14 DOI: https://doi.org/10.2147/IJGM.S301518

[11] Kumar, S., Jha, S., Rai, S.K. (2020). Significance of Super spreader Events in COVID19https://www.researchgate.net/journal/Indian-Journal-of-PublicHealth-0019-557X http://dx.doi.org/10.4103/ijph.IJPH_495_20

[12] Li, D., Wu, S.(2016). Analysis on the health status and influencing factors of medical workers in a city of Fujian province. The Medical Forum 2016;20(14):1893-5. 10

[13] Wang, L. C.X. R., Wu, Y. Zhang, W. L., Tan, X.(2020). Risk factors of healthcare workers with corona virus disease 2019: a retrospective cohort study in a designated hospital of Wuhan in China. Clin Infect Dis. 2020. doi:10.1016/j.cmi.2020.08.038

[14] Pandey. S. K. Sharma, V. (2020).A tribute to frontline corona warriors-Doctors who sacrificed their life while saving patients during the ongoing COVID-19 pandemic. Indian J Ophthalmol. 2020 May; 68(5): 939-942. doi: 10.4103/ijo.IJO_754_20 https://www.ncbi.nlm.nih.gov/pmc/articles/PMC7350477/

[15] Weaver, M.D., Landrigan, C.P., Sullivan, J,P, et al (2020). The association between resident physician work hour regulations and physician safety and health. The American Journal of Medicine 2020

[16] World Health Organization. Health Workers: a global profile, 2020. Available: https://www. who. int/ whr/ 2006/ 06_ chap1_ en. Pdf 